\title{
UNMIXING-BASED DENOISING AS A PRE-PROCESSING STEP FOR CORAL REEF ANALYSIS
}

\author{
D. Cerra ${ }^{\text {a, }}$, D. Traganos ${ }^{\text {b }}$, P. Gege ${ }^{\text {a }}$, P. Reinartz ${ }^{\text {a }}$ \\ ${ }^{a}$ German Aerospace Center (DLR), Earth Observation Center (EOC), 82234 Wessling, Germany \\ (daniele.cerra, peter.gege, peter.reinartz)@dlr.de \\ ${ }^{\mathrm{b}}$ German Aerospace Center (DLR), Remote Sensing Technology Institute, Rutherfordstraße 2, 12489 Berlin, Germany - \\ dimosthenis.traganos@dlr.de
}

Commission I, WG I

KEY WORDS: Hyperspectral remote sensing, Coral, Denoising, Water, Derivative Features, Spectral Unmixing.

\begin{abstract}
:
Coral reefs, among the world's most biodiverse and productive submerged habitats, have faced several mass bleaching events due to climate change during the past 35 years. In the course of this century, global warming and ocean acidification are expected to cause corals to become increasingly rare on reef systems. This will result in a sharp decrease in the biodiversity of reef communities and carbonate reef structures. Coral reefs may be mapped, characterized and monitored through remote sensing. Hyperspectral images in particular excel in being used in coral monitoring, being characterized by very rich spectral information, which results in a strong discrimination power to characterize a target of interest, and separate healthy corals from bleached ones. Being submerged habitats, coral reef systems are difficult to analyse in airborne or satellite images, as relevant information is conveyed in bands in the blue range which exhibit lower signal-to-noise ratio (SNR) with respect to other spectral ranges; furthermore, water is absorbing most of the incident solar radiation, further decreasing the SNR. Derivative features, which are important in coral analysis, result greatly affected by the resulting noise present in relevant spectral bands, justifying the need of new denoising techniques able to keep local spatial and spectral features. In this paper, Unmixing-based Denoising (UBD) is used to enable analysis of a hyperspectral image acquired over a coral reef system in the Red Sea based on derivative features. UBD reconstructs pixelwise a dataset with reduced noise effects, by forcing each spectrum to a linear combination of other reference spectra, exploiting the high dimensionality of hyperspectral datasets. Results show clear enhancements with respect to traditional denoising methods based on spatial and spectral smoothing, facilitating the coral detection task.
\end{abstract}

\section{INTRODUCTION}

Coral reefs, recognized as one of the world's most biodiverse and productive submerged habitats (Connel, 1978), faced 6 mass bleaching events due to climate change and its linked impacts during the past 35 years (Hughes, 2003; Hedley, 2016). Coral bleaching occurs during prolonged periods of increased sea surface temperature (SST) which induce the expulsion of the symbiotic microalgae, zooxanthellae, from the coral host (Glynn, 1990; Atwood, 1992). This phenomenon can rapidly lead to coral mortality and thus substantial decline in biodiversity associated with the reef environment (HoeghGuldberg, 2016). Scientists are predicting increasingly serious consequences for reef-associated fisheries, tourism, coastal protection, and people (Hoegh-Guldberg, 2007). Accurate detection, mapping and modelling of the onset, event and subsequent results of coral bleaching are vital for the longevity of coral reef ecosystems.

Remote sensing is regarded as the most efficient approach to identify coral bleaching events from regional to oceanic basin scale (Yamano, 2004). A major complication in such approaches is the differentiation between the spectral reflectance of bleached coral and that of healthy coral, algae and sand that compose a typical reef environment (Holden, 1999; Clark, 2000). This highlights the importance of high spectral resolution in discriminating the distinct spectral shape of bleached coral (Hochberg, 2004). Furthermore, a high spatial resolution is necessary to map spatial patterns of bleaching, and an optimal Ground Sampling Distance has been suggested in the range 40-80 cm (Andréfouët, 2002). Another crucial aspect is the temporal resolution of the observations: algae, which exhibit similar spectral behavior to zooxanthellae populating healthy coral, can rapidly colonize dead corals following bleaching, thus hindering the detection of the phenomenon (Clark, 2000). Recent development on data acquired by airborne and satellite optical sensors have advanced the identification and mapping of coral bleaching from a theoretical and experimental to a routine level (Hedley, 2016; Holden, 1999; Elvidge, 2004).

Hyperspectral instruments measure the reflected solar energy from a target in up to hundreds of contiguous and narrow spectral bands of the electromagnetic spectrum. The amount of energy reflected in each of the wavelength positions differs depending on the chemical composition of the target material and results in material-specific spectral reflectance signatures, which enable the identification of specific materials. In the case of corals, these sensors excel at mapping the structure of a coral reef and separating healthy from bleached corals.

For these tasks, mostly airborne data have been used in literature, due to the aforementioned need of an adequate GSD, which is not possible to obtain from spaceborne sensors. In the 
specific, derivative features from hyperspectral data convey relevant information for the detection of healthy vs. bleached coral and the characterization of their context (sand bed) (Holden, 2000).

Hyperspectral data also present relevant problematics, hindering their use in some practical applications. Firstly, narrow spectral bands can be affected by a low Signal-to-Noise Ratio (SNR), especially in spectral regions at shorter wavelengths such as the blue portion of the spectrum, where Rayleigh scattering contributes at decreasing the quality of the images, in addition to pronounced atmospheric absorption effects. Furthermore, for applications to submerged objects, the water above absorbs most of the incident solar radiation, further decreasing the SNR and the discrimination capabilities of an acquisition.

It is well known that computing derivative features on noisy data sequences can significantly amplify the noise, hindering such analysis in hyperspectral image processing. As second derivatives are going to be used in this work, multiplying the described effects, efficient image denoising algorithm must be employed in order to be able to extract reliable and consistent derivative features.

Denoising is often carried out in image processing through filtering, usually based on convolutions with sliding windows in the image domain, on operations in the frequency domain, or on estimated noise statistics or degradation functions, if these are known for the image acquisition process (Gonzalez, 2007). In the case of hyperspectral data, the high spectral dimensionality of each image element can be exploited in order to derive a pixel-based denoising exploiting both spatial and spectral information. Unmixing-based Denoising (UBD) is a supervised methodology for the recovery of bands characterized by a low SNR in a hyperspectral scene (Cerra, 2014), which is described in next section.

\section{UNMIXING-BASED DENOISING}

Unmixing-based Denoising (UBD) has been recently proposed to selectively retrieve spectral bands characterised by a low SNR by exploiting their correlation with non-corrupted pixels across the whole spectral dimension in hyperspectral images (Cerra et al., 2014). Spectral unmixing is the process which aims at decomposing each hyperspectral image element as a linear (or less often non-linear) combination of signals typically related to pure materials, often called endmembers, representing the backscattered solar radiation in each spectral band. Considering the physical properties of a mixed spectrum, UBD assumes the residual vector derived from the unmixing process to be mostly composed of noise and more relevant in spectral bands where atmospheric absorption effects are stronger, and therefore ignored in the reconstruction.

These methods give as output abundances maps, which quantify the contribution of each endmember to a given pixel. Therefore, a pixel $m$ could be described as a linear combination of $n$ reference spectra $S=\left\{s_{1}, s_{2}, \ldots, s_{n}\right\}$, weighted by the fractional abundances $X=\left\{x_{1}, x_{2}, \ldots, x_{n}\right\}$, plus a residual vector $r$, containing the portion of the signal which cannot be represented in terms of the basis vectors of choice:

$$
m=\sum_{i=1}^{n} x_{i} s_{i}+r
$$

For example, if in a scene we have only mixtures of two materials in each pixel, for example water and soil, $m$ could be expressed as $m=x_{\text {water }} s_{\text {water }}+x_{\text {soil }} s_{\text {soil }}+r$. The output of the described spectral unmixing process is inferred into the reconstruction of a given noisy band in a hyperspectral dataset through Unmixing-based Denoising (UBD) as follows.

If the modelling errors in $S$ are kept to a minimum, we expect the noise term and local anomalies to be predominant in $r$ for bands with low SNR and corrupted values, respectively. We therefore assume $r$ to be composed by noise, anomalous fluctuation, and artifacts introduced during either the acquisition or the preprocessing step. If $r$ is ignored we can derive a noise-free reconstruction for $m$ which also corrects anomalous values as:

$$
\hat{m}=\sum_{i=1}^{n} x_{i} S_{i}
$$

This means that if the contributions to the radiation reflected from a resolution cell are known, the values of noisy bands can be derived by a combination of the average values characterizing each component in that spectral range. This is done under the assumptions that contributions related to materials not present in S, subtle variations of one or more materials in S, and non-linear mixing effects are negligible.

In spite of adopting a linear unmixing model, which in theory does not adapt well to water in which several non-linear scattering effects take place, UBD achieved satisfactory results when applied to spectral bands in the blue range for scenes acquired over coastal waters in previous works (Cerra et al., 2013). Therein, UBD showed promising results for the estimation of coloured Dissolved Organic Matter (CDOM), and for the regularization of bathymetry estimation in coastal waters.

\section{EXPERIMENTAL RESULTS}

We analyse a hyperspectral scene acquired by the SpecTIR sensor over a coral reef in the Red Sea in Saudi Arabia, of size $960 \times 600$ pixels, with 128 bands acquired in the range 400$1000 \mathrm{~nm}$. The image is processed to top-of-the-atmosphere radiance values. Several corals are visible in the area: Fig. 1 reports a true color combination of the Red Sea image in which these appear in red.

The UBD algorithm does not need the data to be expressed in reflectance, so no conversion from radiance to reflectance has been carried out. We apply the algorithm on the scene by preselecting a spectral library of 15 spectra, chosen according to their Pixel Purity Index (PPI). The spectra should be chosen in order to be as pure as possible: this implies that they should lie on the convex hull encompassing the dataset projected in the space defined by the spectral bands of the image. The PPI helps empirically in selecting pixels as close as possible to the convex hull, increasing the chance of reconstructing any pixel inside the convex hull with limited distortions as quantified by the residual vector $r$ in (1). 


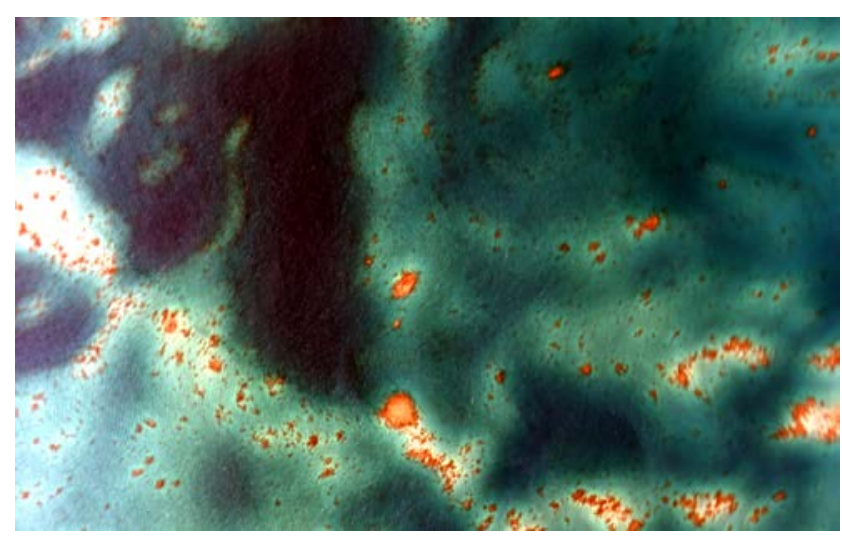

Figure 1. True color combination (central wavelengths 640, 551, and $461 \mathrm{~nm}$ ) from the SpecTIR Red Sea dataset,

$$
\text { (C) } 2012 \text { SpecTIR }
$$

In the following experiments we choose inversion through Nonnegative Least Squares (NNLS) to quantify the abundance related to each reference spectrum in the spectral library, proportional to the area covered by a given material within an image element. It is of interest to remark that NNLS naturally enforces sparsity in its solution, as several components in it are set to zero: this intuitively well agrees with the characteristics of a hyperspectral pixel, which is usually composed by a limited number of materials (Cerra, 2014). Unconstrained Least Squares and first attempts at using sparse reconstruction tools did not yield satisfactory results, while in recent years the fullyconstrained least squares method, which enforces not only nonnegativity but also the sum-to-one constraint on the estimated abundances, has been debated by the community and is therefore not considered in these experiments (Bioucas Dias, 2012).

As a first experiment, the first derivative feature is computed for noisy bands in the blue range. Fig. 2 reports the original derivative and Fig. 3 the result after applying UBD. As the method operates pixelwise, fine details in the image are kept, along with the overall local spectral information.

As a next step, results are assessed on the second derivative feature, which represents a more difficult application. In (Holden, 2000), the authors establish a correlation between the presence of healthy corals and the second derivative feature around $545 \mathrm{~nm}$. Due to the noise present in the image, the computation of a second derivative around $545 \mathrm{~nm}$ results in a very noisy image, in which live corals are very hard to spot (Fig. 4). Even after both a spatial and spectral smoothing of the image prior to the extraction of derivative features, results are hard to interpret (Fig. 5). The pixel-based denoising based on UBD delivers a set of derivative features which appear smooth and correlated to the actual presence of live corals (Fig. 6). Around the live corals, it is easy to identify areas in which the first derivative drops considerably, highlighting areas in which it assumes higher values. All the derivative features reported have undergone the same histogram stretch. In the color mapping used, blue corresponds to low values and red to high ones.

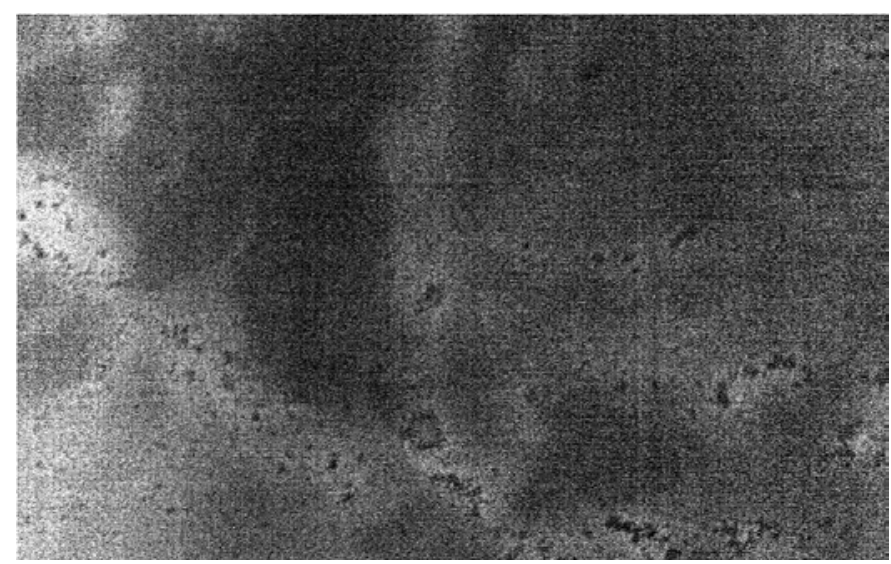

Figure 2. First derivative between spectral bands 3 and 4 of the Red Sea dataset.

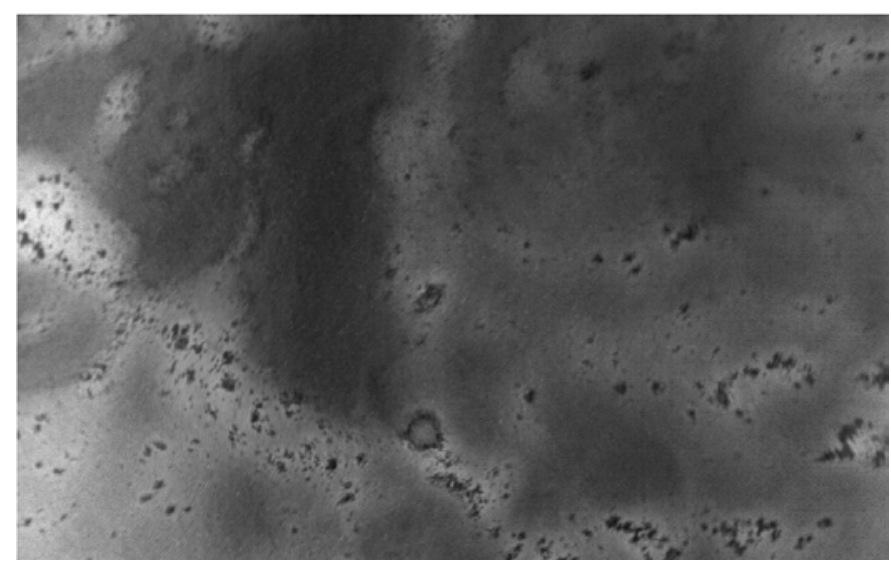

Figure 3. First derivative between spectral bands 3 and 4 of the Red Sea dataset undergone through a denoising step with UBD.

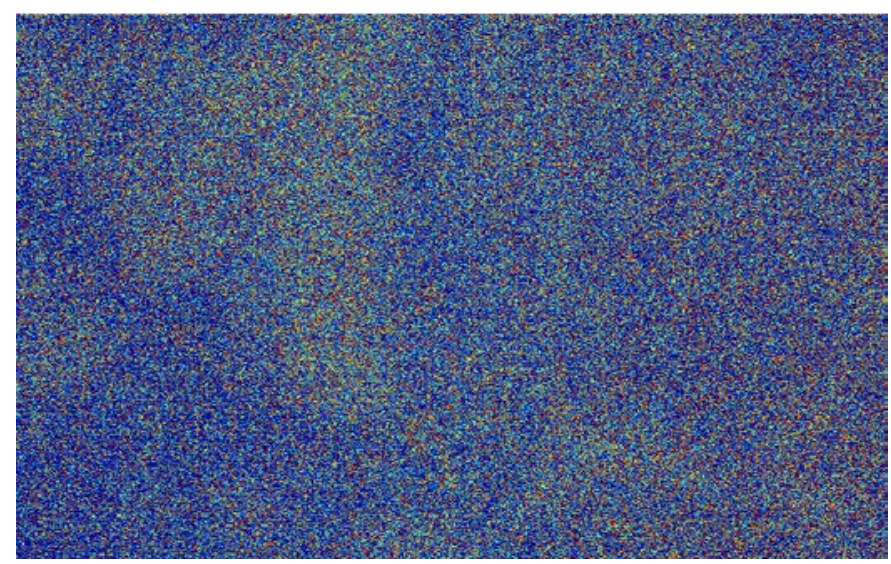

Figure 4. Second derivative at $545 \mathrm{~nm}$ for the Red Sea dataset reported in false colors.

\section{CONCLUSIONS}

Hyperspectral data are powerful at detecting and characterizing coral reefs ecosystems. As derivative features on the spectral information are difficult to use in practice, given the low SNR of bands in the blue range of the spectrum and the low energy 
measured by the sensor which is reflected by the submerged coral reefs, denoising techniques must be employed in order to derive meaningful information from these features.

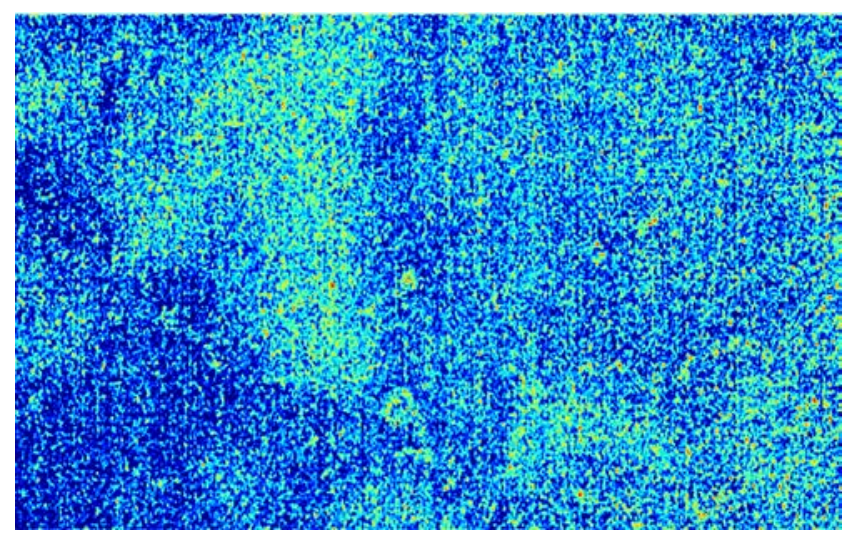

Figure 5. Derivative feature as reported in Fig. 4 computed after a preprocessing step of spatial and spectral smoothing.

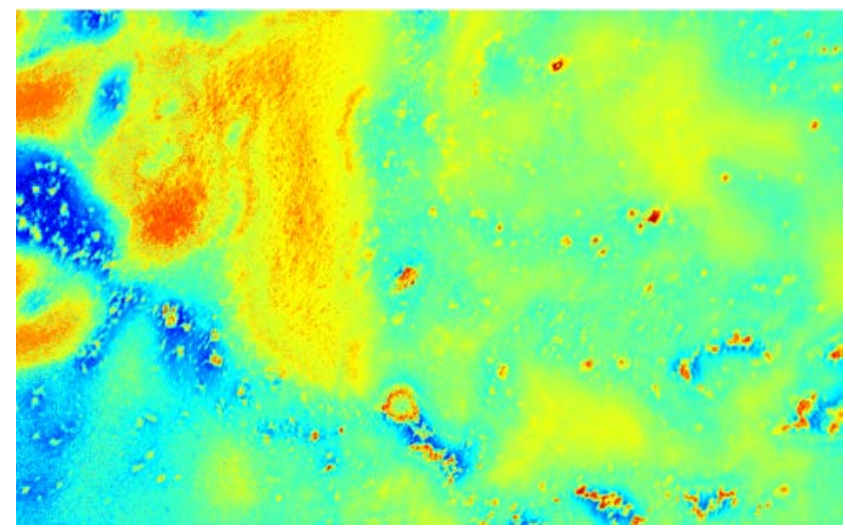

Figure 6. Second derivative at $545 \mathrm{~nm}$ derived after selecting UBD as preprocessing step. The live corals can be identified and match the ones visible in Fig. 1.

Unmixing-based Denoising (UBD) is a supervised methodology for the recovery of bands characterized by a low Signal-toNoise Ratio (SNR) in a hyperspectral scene. UBD reconstructs any pixel in a given band as a linear combination of reference spectra belonging to materials present in the scene. If a perfect unmixing model is given, the residual vector from the unmixing process is mostly composed by contributions of uninteresting materials, unwanted atmospheric influences and sensor-induced noise, and can be thus ignored in the reconstruction process. The reported results are promising and suggest that local information, both spatial and spectral, could be well preserved by selecting UBD as an alternative denoising technique as a pre-processing step for coral reef analysis.

\section{ACKNOWLEDGEMENTS}

The authors thank Benjamin Pröbster for his research on datasets suitable for UBD applications.

\section{REFERENCES}

Andréfouët, S., Berkelmans, R., Odriozola, L., Done, T., Oliver, J. and Müller-Karger, F., 2002, "Choosing the appropriate spatial resolution for monitoring coral bleaching events using remote sensing", Coral Reefs, 21/2, pp. 147-154.
Atwood, D.K., Hendee, J. C., and Mendez, A., 1992, "An Assessment of Global Warming Stress on Caribbean Coral Reef Ecosystems", Bulletin of Marine Science, 51/1, pp. 118-130.

Bioucas Dias, J. M. et al. , 2012, "Hyperspectral Unmixing Overview: Geometrical, Statistical, and Sparse RegressionBased Approaches", IEEE Journal of Selected Topics in Applied Earth Observations and Remote Sensing, 5/2, pp. 354379.

Cerra, D., Müller, R., and Reinartz, P., 2014, "Noise Reduction in Hyperspectral Images Through Spectral Unmixing," IEEE Geoscience and Remote Sensing Letters, 11/1, pp. 109-113.

Cerra, D., Gege, P., Müller, R., and Reinartz, P., 2013, "Exploiting noisy hyperspectral bands for water analysis", 33th EarSEL Symposium Proceedings, pp. 43-48.

Clark, C.D., Mumby, P.J., Chisholm, J.R.M., Jaubert, J., and Andrefouet, S., 2000, "Spectral discrimination of coral mortality states following a severe bleaching event", International Journal of Remote Sensing, 21/11, pp. 23212327.

Connell, J.H., 1978, "Diversity in Tropical Rain Forests and Coral Reefs", Science, 199/4335, pp. 1302-1310.

Elvidge, C.D. et al., 2004, "Satellite observation of Keppel Islands (Great Barrier Reef) 2002 coral bleaching using IKONOS data", Coral Reefs, 23/1, pp. 123-132.

Glynn, P. W. and D'Croz, L., 1990, "Experimental evidence for high temperature stress as the cause of El Niño-coincident coral mortality", Coral Reefs, 8/181, pp. 181-191.\#

Gonzalez, R. C. and Woods, R. E., 2007, "Digital Image Processing ( $3^{\text {rd }}$ Edition)", Prentice Hall.

Hedley, J. et al., 2016, "Remote Sensing of Coral Reefs for Monitoring and Management: A Review,” Remote Sensing, 8/2, p. 118.

Hochberg, E.J., Atkinson, M.J., Andréfouët, S., 2004, "Spectral reflectance of coral reef bottom-types worldwide and implications for coral reef remote sensing", Remote Sensing of Environment, vol. 90, no. 1, pp. 86-103.

Hoegh-Guldberg, B.Y. et al., 2007, "Coral Reefs Under Rapid Climate Change and Ocean Acidification", Science, 318/5857, pp. 1737-1742.

Holden, H. and Ledrew, E., 1999, "Hyperspectral Identification of Coral Reef Features", International Journal of Remote Sensing, 20/13, pp. 2545-2563.

Holden, H. and Ledrew, E., 2000, "Accuracy Assessment of Hyperspectral Classification of Coral Reef Features", Geocarto International, 15/2, pp. 5-11.

Hughes, T. P. et al., 2003, "Climate Change, Human Impacts, and the Resilience of Coral Reefs", Science, 310, pp. 929-933.

Yamano, H. and Tamura, M., 2004, "Detection limits of coral reef bleaching by satellite remote sensing: Simulation and data analysis", Remote Sensing of Environment, 90/1, pp. 86-103. 